\title{
NONDEGENERATE SYMMETRIC BILINEAR FORMS ON FINITE ABELIAN 2-GROUPS
}

BY

RICK MIRANDA ${ }^{1}$

\begin{abstract}
Let $\mathscr{B}_{2}$ be the semigroup of isomorphism classes of finite abelian 2-groups with a nondegenerate symmetric bilinear form having values in $Q / \mathbf{Z}$. Generators for $\mathscr{B}_{2}$ were given by C. T. C. Wall and the known relations among these generators were proved to be complete by A. Kawauchi and S. Kojima. In this article we describe a normal form for such bilinear forms, expressed in terms of Wall's generators, and as a by-product we obtain a simpler proof of the completeness of the known relations.
\end{abstract}

1. Introduction. Let $G$ be a finite abelian group. A symmetric bilinear form on $G$ is a map $b: G \times G \rightarrow Q / Z$ such that $b(x, y)=b(y, x)$ and $b(x,-)$ is a group homomorphism from $G$ to $Q / \mathbf{Z}$ for every $x$ and $y$ in $G$. The form $b$ is nondegenerate if $b(x,-)$ is not the trivial homomorphism for $x \neq 0$. Let $\mathscr{B}$ be the semigroup of isomorphism classes of nondegenerate symmetric bilinear forms on finite abelian groups, under orthogonal direct sum. It is not hard to see that any such form will split orthogonally into forms on the Sylow $p$-subgroups, so $\mathscr{B}$ is canonically isomorphic to $\oplus \mathscr{B}_{p}$, where $\mathscr{B}_{p}$ is the semigroup of such forms on finite abelian p-groups.

Wall [2] has shown that if $p$ is odd, $\mathscr{B}_{p}$ is generated by forms on cyclic groups. If $G=\mathbf{Z} / p^{r}$, he denotes by $A_{p^{r}}$ the form $b$ with $b(1,1)=1 / p^{r}$, and by $B_{p^{r}}$ the form $b$ with $b(1,1)=n / p^{r}$ with $n$ a nonsquare $\bmod p$. Wall's theorem is that $\mathscr{B}_{p}$ is generated by $\left\{A_{p^{r}}, B_{p^{r}} ; r \geqslant 1\right\}$ with the relations $2 A_{p^{r}}=2 B_{p^{r}}$ for each $r$ when $p$ is odd.

The generators and relations for $p=2$ are quite a bit more complicated and in his paper Wall only gave the following set of generators of $\mathscr{B}_{2}$ :

$$
\begin{aligned}
& A_{2^{r}} \quad \text { on } \mathbf{Z} / 2^{r}, r \geqslant 1 ; b(1,1)=1 / 2^{r}, \\
& B_{2^{r}} \quad \text { on } \mathbf{Z} / 2^{r}, r \geqslant 2 ; b(1,1)=-1 / 2^{r}, \\
& C_{2^{r}} \quad \text { on } \mathbf{Z} / 2^{r}, r \geqslant 3 ; b(1,1)=5 / 2^{r}, \\
& D_{2^{r}} \quad \text { on } \mathbf{Z} / 2^{r}, r \geqslant 3 ; b(1,1)=-5 / 2^{r}, \\
& E_{2^{r}} \quad \text { on } \mathbf{Z} / 2^{r} \times \mathbf{Z} / 2^{r}, r \geqslant 1 ; b\left(e_{i}, e_{j}\right)= \begin{cases}0 & \text { if } i=j, \\
1 / 2^{r} & \text { if } i \neq j,\end{cases}
\end{aligned}
$$

Received by the editors May 23, 1983.

1980 Mathematics Subject Classification. Primary 10C05, 15A63.

${ }^{1}$ Partially supported by the NSF. 


$$
F_{2^{r}} \quad \text { on } \mathbf{Z} / 2^{r} \times \mathbf{Z} / 2^{r}, r \geqslant 2 ; b\left(e_{i}, e_{j}\right)= \begin{cases}1 / 2^{r-1} & \text { if } i=j, \\ 1 / 2^{r} & \text { if } i \neq j,\end{cases}
$$

where $\left\{e_{1}, e_{2}\right\}$ generate $\mathbf{Z} / 2^{r} \times \mathbf{Z} / 2^{r}$ in the last two cases.

The remaining problem is to determine all the relations among the above generators for $\mathscr{B}_{2}$, which Wall declined to do in his article. This problem was solved completely by Kawauchi and Kojima [1], who not only proved that a certain list of relations was complete, but also gave a complete system of invariants which distinguished between any two nonisomorphic forms.

It is the purpose of this article to present a proof of Kawauchi and Kojima's theorem which is simpler and more straightforward than the original. As a by-product, a normal form for any nondegenerate symmetric bilinear form on a finite abelian 2-group is obtained and an algorithm is presented to put an arbitrary form into the normal form.

Since I will be only concerned with 2-groups in this article, I will use the notation $A_{r}$ for $A_{2^{r}}$, and similarly for the other forms; in addition, I will introduce the forms $B_{1}=C_{1}=D_{1}\left(=A_{1}\right), C_{2}\left(=A_{2}\right), D_{2}\left(=B_{2}\right)$ and $F_{1}\left(=E_{1}\right)$ for convenience. Finally, I will use the word form to denote a nondegenerate symmetric bilinear form.

The main theorem can now be stated.

THEOREM 1.1 [1, TheOREM 0.1]. The semigroup $B_{2}$ is generated by $\left\{A_{r}, B_{r}, C_{r}, D_{r}\right.$, $\left.E_{r}, F_{r} ; r \geqslant 1\right\}$ and any relation among them is generated by the relations in the following table.

TABLE 1.2

Relations among the generators of $B_{2}$

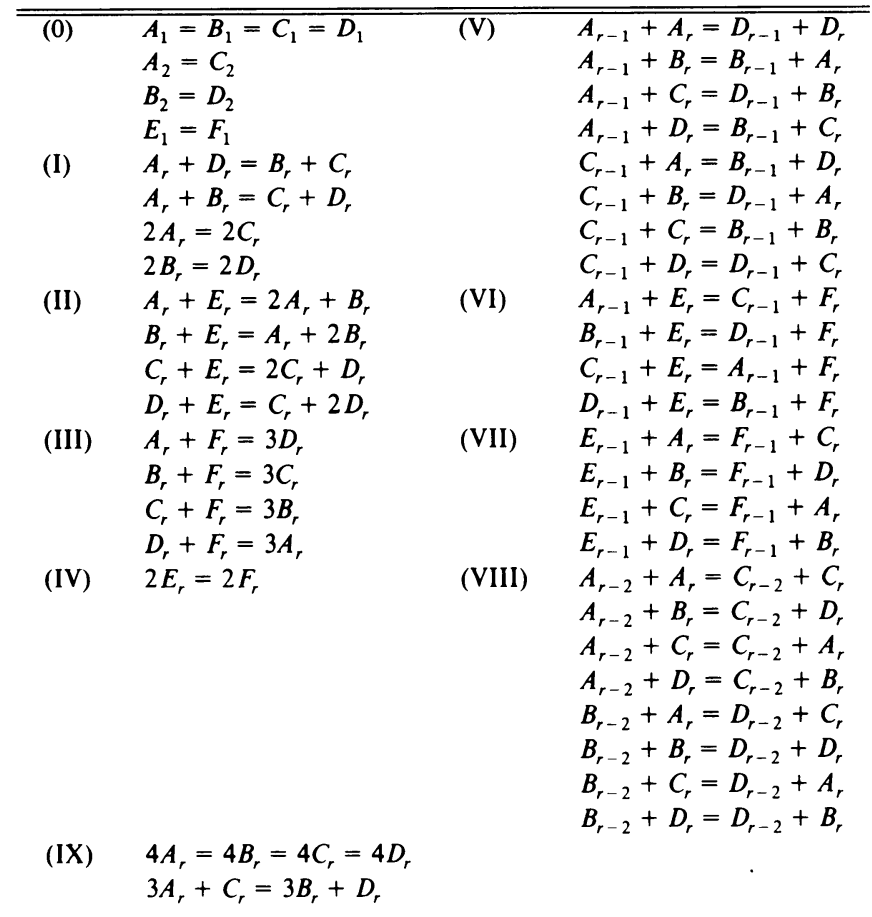


The relations in (IX) are consequences of those in (I), (II) and (III), so I have put them last; they are useful in determining the normal forms for homogeneous groups.

The reader may verify that the relations in Table 1.2 do hold; it is our intent only to verify their completeness.

2. The signature invariants. Let $G$ be a finite abelian 2-group. Let $G_{k}=\{x \in$ $\left.G \mid 2^{k} x=0\right\} ; G_{k}$ is a subgroup of $G, G_{0}=(0), G_{k} \subseteq G_{k+1}$ for every $k$, and $G_{k}=G$ for $k$ sufficiently large. Define $\rho_{k}(G)=G_{k} /\left(G_{k-1}+2 G_{k+1}\right)$. This group is either 0 or is a 2-group with exponent 1 ; if $G \cong \oplus_{k}\left(\mathbf{Z} / 2^{k}\right)^{r(k)}$, then $\rho_{k}(G) \cong(\mathbf{Z} / 2)^{r(k)}$. Let $b$ be a form on $G$. Then $b$ induces a form $b_{k}$ on $\rho_{k}(G)$, defined by

$$
b_{k}(\bar{x}, \bar{y})=2^{k-1} b(x, y),
$$

where $x$ and $y$ are in $G_{k}$ and $\bar{x}, \bar{y}$ are their images in $\rho_{k}(G)$.

Using the relation $A_{1}+E_{1}=3 A_{1}$, one can easily see that any form $b$ on $(\mathbf{Z} / 2)^{N}$ is isomorphic to either $N A_{1}$ or $(N / 2) E_{1}$, the latter occurring only if $N$ is even. These are nonisomorphic: every element $x$ of $(N / 2) E_{1}$ satisfies $b(x, x)=0$, which is not the case for $N A_{1}$. We will say that $b$ is of type $A$ or of type $E$, respectively.

Let $b$ be a form on an arbitrary 2-group $G$ and assume that $b_{k}$ is of type $E$. In this case the function $q_{k}: G / G_{k} \rightarrow Q / \mathbf{Z}$ defined by $q_{k}(\bar{x})=2^{k-1} b(x, x)$ is well defined, where $x$ is any preimage in $G$ of $\bar{x}$ in $G / G_{k}$. Let

$$
S_{k}(b)=\frac{\left|G_{k}\right|^{1 / 2}}{|G|^{1 / 2}} \sum_{x \in G / G_{k}} \exp \left(2 \pi i q_{k}(x)\right)
$$

LeMma 2.1. If $b_{k}$ is of type $E$, then $S_{k}(b)$ is a complex eighth root of unity.

Proof. See [1, Lemma 2.1 and Corollary 2.1].

Kawauchi and Kojima's signature invariant can now be defined. Let $\overline{\mathbf{Z}}_{8}=\mathbf{Z} / 8 \mathrm{U}$ $\{\infty\}$ be the semigroup with 9 elements $0, \ldots, 7, \infty$ whose operation is defined by addition mod 8 within $\mathbf{Z} / 8$ and $i+\infty=\infty+i=\infty+\infty=\infty$.

Definition 2.2. Let $b$ be a form on $G$. If $b_{k}$ is of type $E$, let $\sigma_{k}(b)$ be the element of $\mathbf{Z} / 8$ such that $S_{k}(b)=\exp \left(\pi i \sigma_{k}(b) / 4\right)$. If $b_{k}$ is of type $A$, let $\sigma_{k}(b)=\infty$.

Hence $\sigma_{k}(b)$ is an element of $\overline{\mathbf{Z}}_{8}$ for any $k$.

LEMMA 2.3. (1) If $b$ and $b^{\prime}$ are forms on $G$ and $G^{\prime}$, respectively, then $\left(b, b^{\prime}\right)$ is a form on $G \oplus G^{\prime}$ and $\sigma_{k}\left(b, b^{\prime}\right)=\sigma_{k}(b)+\sigma_{k}\left(b^{\prime}\right)$ for every $k$.

(2) If $G \cong\left(\mathbf{Z} / 2^{r}\right)^{N}$ and $b$ is a form on $G$, then $\sigma_{k}(b)=0$ for $k>r$. 1.

(3) $\sigma_{k}\left(A_{k}\right)=\sigma_{k}\left(B_{k}\right)=\sigma_{k}\left(C_{k}\right)=\sigma_{k}\left(D_{k}\right)=\infty$ and $\sigma_{k}\left(E_{k}\right)=\sigma_{k}\left(F_{k}\right)=0$ for $k \geqslant$

(4) $\sigma_{k-1}\left(A_{k}\right)=\sigma_{k-1}\left(C_{k}\right)=1, \quad \sigma_{k-1}\left(B_{k}\right)=\sigma_{k-1}\left(D_{k}\right)=7, \quad \sigma_{k-1}\left(E_{k}\right)=0$, and $\sigma_{k-1}\left(F_{k}\right)=4$ for $k \geqslant 2$.

(5) $\sigma_{k-2}\left(A_{k}\right)=1, \sigma_{k-2}\left(B_{k}\right)=7, \sigma_{k-2}\left(C_{k}\right)=5, \sigma_{k-2}\left(D_{k}\right)=3$, and $\sigma_{k-2}\left(E_{k}\right)=$ $\sigma_{k-2}\left(F_{k}\right)=0$ for $k \geqslant 3$.

Proof. See [1, Lemma 2.1 and Corollary 2.2]. 
3. Normal forms for homogeneous groups. The group $G$ is homogeneous if $G \cong$ $\left(\mathbf{Z} / 2^{r}\right)^{N} ; r$ is the exponent of $G$ and $N$ is the rank of $G$. In this section I will present normal forms for forms on homogeneous 2-groups, which differ from those given in [1].

Definition 3.1. Let $b$ be a form on a homogeneous 2-group. The form $b$ is of type $A$ if it is isomorphic to $N A_{r}$. It is of type $B$ if the form can be written in terms of $A_{r}$ and $B_{r}$. It is of type $C$ if it can be written in terms of $A_{r}$ and $C_{r}$. It is of type $D$ if it can be written in terms of $A_{r}, B_{r}, C_{r}$ and $D_{r}$. It is of type $E$ if it is isomorphic to $M E_{r}$, where $2 M$ is the rank of the group, and it is of type $F$ if it can be written in terms of $E_{r}$ and $F_{r}$.

This terminology agrees with the previously introduced notion of type for exponent-one groups. It is easy to see (using the relations (II) and (III) of Table 1.2) that every form on a homogeneous group has a type. However, some forms may be of more than one type. (For example, every form of type $E$ is of type $F$.)

In Table 3.2 the desired normal forms for forms on homogeneous 2-groups are given, together with their types and the three most useful signature invariants.

TABLE 3.2

Normal forms for forms on homogeneous 2-groups of exponent $r$ and rank $N$

\begin{tabular}{|c|c|c|c|c|c|c|}
\hline exponent $r$ & $\operatorname{rank} N$ & normal form & $\sigma_{r}$ & $\sigma_{r-1}$ & $\sigma_{r-2}$ & type \\
\hline 1 & $N \geqslant 1$ & $\overline{N A_{1}}$ & $\infty$ & --.- & 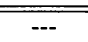 & $\bar{A}$ \\
\hline 1 & $N$ even & $(N / 2) E_{1}$ & 0 & --- & --- & $E$ \\
\hline 2 & $N \geqslant 1$ & $\mathrm{NA}_{2}$ & $\infty$ & $N$ & --- & $A$ \\
\hline 2 & $N \geqslant 1$ & $(N-1) A_{2}+B_{2}$ & $\infty$ & $N+6$ & -- & $B$ \\
\hline 2 & $N \geqslant 2$ & $(N-2) A_{2}+2 B_{2}$ & $\infty$ & $N+4$ & --- & $B$ \\
\hline 2 & $N \geqslant 3$ & $(N-3) A_{2}+3 B_{2}$ & $\infty$ & $N+2$ & --- & $B$ \\
\hline 2 & $N$ even & $(N / 2) E_{2}$ & 0 & 0 & --- & $E$ \\
\hline 2 & $N$ even & $((N / 2)-1) E_{2}+F_{2}$ & 0 & 4 & --- & $F$ \\
\hline$r \geqslant 3$ & 1 & $D_{r}$ & $\infty$ & 7 & 3 & $D$ \\
\hline " & 2 & $B_{r}+D_{r}$ & $\infty$ & 6 & 2 & $D$ \\
\hline " & 3 & $2 B_{r}+D_{r}$ & $\infty$ & 5 & 1 & $D$ \\
\hline$"$ & $N \geqslant 1$ & $(N-1) A_{r}+C_{r}$ & $\infty$ & $N$ & $N+4$ & C \\
\hline ” & $N \geqslant 2$ & $(N-2) A_{r}+B_{r}+C_{r}$ & $\infty$ & $N+6$ & $N+2$ & $D$ \\
\hline " & $N \geqslant 3$ & $(N-3) A_{r}+2 B_{r}+C_{r}$ & $\infty$ & $N+4$ & $N$ & $D$ \\
\hline " & $N \geqslant 4$ & $(N-4) A_{r}+3 B_{r}+C_{r}$ & $\infty$ & $N+2$ & $N+6$ & $D$ \\
\hline$"$ & $N \geqslant 1$ & $N A_{r}$ & $\infty$ & $N$ & $N$ & $A$ \\
\hline$"$ & $N \geqslant 1$ & $(N-1) A_{r}+B_{r}$ & $\infty$ & $N+6$ & $N+6$ & $B$ \\
\hline$”$ & $N \geqslant 2$ & $(N-2) A_{r}+2 B_{r}$ & $\infty$ & $N+4$ & $N+4$ & $B$ \\
\hline ” & $N \geqslant 3$ & $(N-3) A_{r}+3 B_{r}$ & $\infty$ & $N+2$ & $N+2$ & $B$ \\
\hline " & $N$ even & $(N / 2) E_{r}$ & 0 & 0 & 0 & $E$ \\
\hline " & $N$ even & $((N / 2)-1) E_{r}+F_{r}$ & 0 & 4 & 0 & $F$ \\
\hline
\end{tabular}

That any form may be brought to one of the above forms is an immediate consequence of the relations (0), (I), (II), (III), (IV) and (IX). An inspection of the above signature invariants shows that the normal forms above are all distinct.

4. Normal forms for forms on an arbitrary 2-group. Given a form $b$ on an arbitrary finite abelian 2-group $G$, we may write $b=\oplus b(r)$, where $b(r)$ is a form on a homogeneous 2-group of exponent $r$. Each $b(r)$ may be independently put into a 
normal form as in the previous section, using the relations (0)-(IV). In this section I will describe how to use the relations (V), (VI), (VII) and (VIII) to put the entire form $b$ into a normal form. For what follows, I will say that the trivial group is of type 0 .

The algorithm to put $b(r)$ into normal form works inductively from above. The key step is to put $b(r)$ into normal form by using the relations of Table 1.2 and to do so without affecting $b(s)$, unless $s=r-2$ or $r-1$. Of course, if $b(r)$ is of type 0 , there is nothing to do, so I will assume that this is not the case.

Table 4.1 gives an algorithm for the first step in this process. The first three columns contain the type of $\rho_{r-2}(G), \rho_{r-1}(G)$ and $\rho_{r}(G)$, and should be regarded as the input to the algorithm. In the fourth column is the type in which $b(r)$ may be put, using the relations in the fifth column (together with the relations (0)-(IV)).

\begin{tabular}{ccccc}
\multicolumn{5}{c}{ TABLE 4.1 } \\
$\rho_{r-2}(G)$ & $\rho_{r-1}(G)$ & $\rho_{r}(G)$ & type of $b(r)$ & relations used \\
\hline \hline $0, A$, or $E$ & 0 or $E$ & $E$ & $F$ & -- \\
$0, A$, or $E$ & $A$ & $E$ & $E$ & $(\mathrm{VI})$ \\
0 or $E$ & 0 & $A$ & $D$ & --- \\
$A$ & 0 & $A$ & $B$ & (VIII) \\
0 or $E$ & $E$ & $A$ & $B$ & (VII) \\
$A$ & $E$ & $A$ & $B$ & (VII) or (VIII) \\
0 or $E$ & $A$ & $A$ & $C$ & (V) \\
$A$ & $A$ & $A$ & $A$ & (V) and (VIII)
\end{tabular}

The verification of Table 4.1 is easy, given the following observation. Note that $\rho_{k}\left(A_{k}\right)=\rho_{k}\left(B_{k}\right)=\rho_{k}\left(C_{k}\right)=\rho_{k}\left(D_{k}\right)=A_{1}$ and $\rho_{k}\left(E_{k}\right)=\rho_{k}\left(F_{k}\right)=E_{1}$. Therefore, if $\rho_{k}(G)$ is of type $A$ there must exist a summand in $b(k)$ of the form $A_{k}, B_{k}, C_{k}$ or $D_{k}$ in any decomposition of $b$ and $b(k)$. Similarly, if $\rho_{k}(G)$ is of type $E, b(k)$ must be of type $F$ (i.e., every summand is $E_{k}$ or $F_{k}$ ) in every decomposition of $b$ and $b(k)$.

Using this remark, I will check Table 4.1 in one representative case, leaving the rest to the reader. Let us examine the second line of the table, where $\rho_{r}(G)$ is of type $E$ and $\rho_{r-1}(G)$ is of type $A$. By the above, $b(r-1)$ must contain one of $A_{r-1}, B_{r-1}, C_{r-1}$ or $D_{r-1}$ as a direct summand. Moreover, by Table 3.2, $b(r)$ may be put in the form $M E_{r}$ or $(M-1) E_{r}+F_{r}$, where $2 M$ is the rank of $b(r)$. If $b(r)=M E_{r}$, there is nothing to do; $b(r)$ is already of type $E$. If $b(r)=(M-1) E_{r}$ $+F_{r}$, then as the table indicates, we may use one of the relations of (VI) to change the $F_{r}$ to an $E_{r}$ since one of $A_{r-1}, B_{r-1}, C_{r-1}$, or $D_{r-1}$ is present. This verifies the second line of Table 4.1 and illustrates the general argument.

If one decrees that $\rho_{-2}(G)$ and $\rho_{-1}(G)$ are of type $A$, then Table 4.1 applies where $r=1$ and $r=2$, also.

We will say that an expression for $b$ in terms of the generators $A_{r}, B_{r}, C_{r}, D_{r}, E_{r}$ and $F_{r}$ is in intermediate normal form if each $b(r)$ is in one of the normal forms of Table 3.2 and the type of $b(r)$ is consistent with the types of $\rho_{r-2}(G), \rho_{r-1}(G)$ and $\rho_{r}(G)$ as determined by Table 4.1 .

This is only an intermediate normal form because there is one other set of relations which can be deduced from those in (V) and which can be further applied. 
The situation in which they come to play is that of the second-to-last line of Table 4.1, i.e., $\rho_{r}(G)$ and $\rho_{r-1}(G)$ are of type $A$ and $\rho_{r-2}(G)$ is of type 0 or type $E$. In this case one can use the relations of (V) to change $B_{r}$ 's and $D_{r}$ 's to $A_{r}$ 's and $C_{r}$ 's in $b(r)$ and achieve a type $C$ form for $b(r)$. Note that then $b(r)$ must equal $N A_{r}$ or $(N-1) A_{r}+C_{r}$ by Table 3.2; this is a consequence of the relation $2 C_{r}=2 A_{r}$.

It is possible in certain circumstances to put $b(r)$ into type $A$ form in this case, i.e., change the $C_{r}$ to an $A_{r}$. For this one uses the following set of relations:

$$
\begin{aligned}
2 A_{r-1}+C_{r} & =B_{r-1}+D_{r-1}+A_{r} \\
2 C_{r-1}+C_{r} & =B_{r-1}+D_{r-1}+A_{r} \\
A_{r-1}+C_{r-1}+C_{r} & =2 B_{r-1}+A_{r} \\
2 B_{r-1}+C_{r} & =A_{r-1}+C_{r-1}+A_{r} \\
2 D_{r-1}+C_{r} & =A_{r-1}+C_{r-1}+A_{r} \\
B_{r-1}+D_{r-1}+C_{r} & =2 A_{r-1}+A_{r} .
\end{aligned}
$$

Each of these relations can be obtained from two of the relations in (V), used successively. For example, $2 A_{r-1}+C_{r}=A_{r-1}+D_{r-1}+B_{r}=B_{r-1}+D_{r-1}+A_{r}$. I will leave the rest to the reader. Using these relations, we have the following.

LEMMA 4.2. Assume $b$ is in intermediate form with $\rho_{r}(G)$ and $\rho_{r-1}(G)$ of type $A$, and $\rho_{r-2}(G)$ of type 0 or type $E$. Then $b(r)$ may be put into type $A$ form, i.e., $b(r)=N A_{r}$, if either:

(a) the rank of $b(r-1) \geqslant 3$, or

(b) the rank of $b(r-1)=2$ and $\sigma_{r-2}(b(r-1)) \neq 0$, i.e., $b(r-1)$ is one of the six types $2 A_{r-1}, A_{r-1}+C_{r-1}, 2 C_{r-1}, 2 B_{r-1}, B_{r-1}+D_{r-1}$, or $2 D_{r-1}$.

PROof. Part (b) follows from the relation of (X) and a computation of $\rho_{r-2}$ for the 10 possible rank-two type $D$ exponent $r-1$ forms. To prove (a), it suffices to show that if the rank of $b(r-1) \geqslant 3$, then one of the six types which can be used in (X) must occur in $b(r-1)$. Since $b(r-1)$ is in normal form, this follows from an inspection of Table 3.2. Q.E.D.

Definition 4.3. An expression for $b$ is in normal form if it is in intermediate form and if the hypotheses of Lemma 4.2 are satisfied for $b$ for any $r$, then $b(r)$ is in type $A$ form.

By Table 4.1 and Lemma 4.2, every form on a finite abelian 2-group $G$ may be put into a normal form by using the relations of $(0)-($ VIII). The main result can now be proved.

THEOREM 4.4. Let $G$ be a finite abelian 2-group. Let $b$ be a nondegenerate symmetric bilinear form on $G$. Then $b$ has only one expression as a direct sum of the generators of $\mathscr{B}_{2}$ which is in normal form.

Proof. Let $N(r)$ be the rank of $\rho_{r}(G)$, so that $G \cong \oplus\left(\mathbf{Z} / 2^{r}\right)^{N(r)}$. I will show that the numbers $N(r)$ and the elements $\sigma_{r}(b) \in \overline{\mathbf{Z}}_{8}$ determine the normal form for $b$. The normal form will be developed by descending induction on $r$; since $N(r)=0$ 
for $r$ large enough, the induction may begin without any analysis. Let us then assume that for $k>r$, it has been shown that there is only one expression for $b$ in exponent $k$, which is in normal form. It will suffice to show that the normal form expression for the exponent $r$ piece of $b$ is determined. There are several cases to consider. Write $\sigma_{r}$ for $\sigma_{r}(b)$ for every $r \geqslant 1$ and $b(r)$ for the exponent $r$ piece of $b$. We may assume $N(r) \geqslant 1$.

Case 1. $\sigma_{r} \neq \infty$. Here, by Table 3.2, the normal form for the exponent $r$ piece of $b$ is written in terms of $E_{r}$ and $F_{r}$, so $\rho_{r}(G)$ is of type $E$. If $\sigma_{r-1}(G)=\infty$, then $\rho_{r-1}(G)$ is of type $A$ and Table 4.1 requires $b(r)$ to be of type $E$, which forces $b(r)=$ $(N(r) / 2) E_{r}$. If $\sigma_{r-1}(G) \neq \infty$, then $\rho_{r-1}(G)$ is of type 0 or type $E$, so that any expression for $b(r-1)$ must be of type $F$, and the contribution to $\sigma_{r-1}$ from $b(r-1)$ is zero by Table 3.2. Since $b(k)$ is determined for $k>r, \sigma_{r-1}$ determines $b(r)$ in this case; the two possibilities $(N(r) / 2) E_{r}$ and $((N(r) / 2)-1) E_{r}+F_{r}$ have $\sigma_{r-1}$ differing by 4 .

Case 2. $\sigma_{r}=\infty$. By Table 3.2, the normal form expression for $b(r)$ is written in terms of $A_{r}, B_{r}, C_{r}$ and $D_{r}$, so that $\rho_{r}(G)$ is of type $A$. There are several subcases to consider.

Case 2A. $\sigma_{r-2}=\sigma_{r-1}=\infty$. Here, by Table $4.1, b(r)$ is of type $A$ so that $b(r)=N(r) A_{r}$ is its normal form.

Case 2B. $\sigma_{r-2}=\infty, \sigma_{r-1} \neq \infty$. In this case $\rho_{r-2}(G)$ is of type $A$ and $\rho_{r-1}(G)$ is of type 0 or $E$, so that by Table 4.1 the normal form for $b(r)$ is of type $B$ and is, in fact, $b(r)=(N(r)-i) A_{r}+i B_{r}$ for some $i$ between 0 and 3. Again the contribution from $b(r-1)$ to $\sigma_{r-1}$ is zero, and by Table $3.2 \sigma_{r-1}$ distinguishes between the above four possibilities, so $b(r)$ is determined.

Case 2C. $\sigma_{r-2} \neq \infty, \sigma_{r-1} \neq \infty$. If $N(r-1)=0$ so that $\rho_{r-1}(G)$ is of type 0 , then (since in this case $\rho_{r-2}(G)$ is of type 0 or $E$ ), Table 4.1 implies that nothing can be done to alter $b(r)$, and any of the normal forms of Table 3.2 are possible. In this case there is no contribution to either $\sigma_{r-2}$ or $\sigma_{r-1}$ from $b(r-2)$ and $b(r-1)$; moreover, by Table 3.2, these invariants distinguish between all of the normal forms with $\sigma_{r}=\infty$. Hence $b(r)$ is determined.

If $N(r-1) \geqslant 1$, then $\rho_{r-1}(G)$ is of type $E$, so by Table 4.1 the normal form for $b(r)$ is of type $B$. Here the argument is as in Case 2B; $\sigma_{r-1}$ distinguishes between the four possibilities for $b(r)$, so it is determined.

Case 2D. $\sigma_{r-2} \neq \infty, \sigma_{r-1}=\infty$. In this case $\rho_{r-2}(G)$ is of type 0 or $E$ and $\rho_{r-1}(G)$ is of type $A$, so by Table 4.1 the intermediate normal form for $b(r)$ is of type $C$. Put $b(r-1)$ into a normal form. If $N(r-1) \geqslant 3$ or $N(r-1)=2$ and $\sigma_{r-2}(b(r-1))$ $\neq 0$, then Lemma 4.2 applies and the normal form for $b(r)$ is of type $A$, so that $b(r)=N(r) A_{r}$. Assume $N(r-1)=2$ but $\sigma_{r-2}(b(r-1))=0$. Then there is no contribution to $\sigma_{r-2}$ from either $b(r-2)$ or $b(r-1)$; since the two possibilities $N(r) A_{r}$ and $(N(r)-1) A_{r}+C_{r}$ are distinguished by $\sigma_{r-2}$ by Table $3.2, b(r)$ is determined. Finally, assume $N(r-1)=1$. Again there is no contribution to $\sigma_{r-2}$ from $b(r-2)$. The two possibilities for $b(r)$ yield a difference of 4 in $\sigma_{r-2}$; if they are both possible, then this difference of 4 must be offset by a difference of 4 contributed from $b(r-1)$. However, $N(r-1)=1$, so that the possibilities for $b(r-1)$ are $A_{r-1}, B_{r-1}, C_{r-1}$ or $D_{r-1}$ and by Lemma 2.3(4), their contributions to 
$\sigma_{r-2}$ are either 1 or 7 . Hence a difference of 4 cannot be contributed by $b(r-1)$, and $b(r)$ is again determined. Q.E.D.

Corollary 4.5 [1, Theorem 4.1]. Any two forms $b$ and $b^{\prime}$ with the same signature invariants $\sigma_{r}$ and ranks $N(r)$ for every exponent $r$ are isomorphic.

Proof. From the proof of the previous theorem, the normal forms for $b$ and $b^{\prime}$ are determined by the invariants $\sigma_{r}$ and the rank $N(r)$. Since these normal forms are identical, $b$ and $b^{\prime}$ are isomorphic. Q.E.D.

COROLLARY 4.6. The relations of Table 1.2 are complete.

Proof. Assume there is a relation of the form $b \cong b^{\prime}$, where $b$ and $b^{\prime}$ are expressed in terms of the given generators for $\mathscr{B}_{2}$. By using the relations of Table 1.2 , both $b$ and $b^{\prime}$ may be brought to normal form. By Theorem 4.4, these normal forms must be identical. By reversing this procedure, the relation $b \cong b^{\prime}$ is obtained from those of Table 1.2. Q.E.D.

\section{REFERENCES}

1. A Kawauchi and S. Kojima, Algebraic classification of linking pairings on 3-manifolds, Math. Ann. 253 (1980), 29-42.

2. C. T. C. Wall, Quadratic forms on finite groups, and related topics, Topology 2 (1964), 281-298.

Department of Mathematics, Colorado State University, Fort Collins, Colorado 80523

Current address: Department of Mathematics, Tufts University, Medford, Massachusetts 02155 